\title{
FORUM
}

\section{Auf ein Neues? Erfolgsfaktoren für die Konferenz zur Zukunft Europas}

\author{
Joachim Wuermeling*
}

In this article, the author contrasts the experience of the Convention on the Future of Europe 20022003 with the mandate for the Conference on the Future of Europe which commenced on May 9, 2021. He criticises that the Conference is deprived of fundamental structural elements that were success factors for the Convention: a clear mandate, a tight leadership, and the right to self-organise. Nevertheless, the author is convinced that the Conference still has every chance of giving the European Union a forward-looking impetus for reform. He derives five recommendations for the future work of the Conference. In particular, it must be set up considering the desired outcome, by defining a product that is to be established, and directing the work toward its creation. If the Conference serves solely as a sounding board for diffuse citizen concerns, its potential cannot be fully exploited.

„Sie muss gut vorbereitet werden: mit einem klar abgesteckten Rahmen und eindeutigen Zielen“, so beschrieb die Präsidentin der Europäischen Kommission, Ursula von der Leyen, die erstrebte Startposition für die Konferenz zur Zukunft Europas (im Weiteren: die Konferenz). ${ }^{1}$ Davon ist gegenwärtig wenig zu sehen, obwohl die Konferenz bereits am 9. Mai 2021 ihre Arbeit aufgenommen hat. Die von Parlament, Kommission und Rat dazu beschlossene Gemeinsame Erklärung ${ }^{2}$ vom 1. März 2021 bleibt in vielerlei Hinsicht vage.

Nicht anders verhielt es sich vor dem Konvent zur Zukunft Europas (im Weiteren: der Konvent), der mit der Erklärung von Laeken vom 15. Dezember 2001 eingesetzt worden war. Dem Konvent, so hieß es damals, falle lediglich die Aufgabe zu, „die wesentlichen Fragen zu prüfen, welche die künftige Entwicklung der Union aufwirft, und sich um verschiedene mögliche Antworten zu bemühen“. 3 Dennoch legte dieser Konvent nach 17 Monaten einen vollständig ausformulierten Entwurf für einen europäischen Verfassungsvertrag vor. ${ }^{4}$ Zwar wurde der Text nicht ratifiziert. Als Erfolg darf der Konvent aber dennoch bezeichnet werden. Der Entwurf wurde in den Volksabstimmungen in Frankreich und den Niederlan-

\footnotetext{
* Prof. Dr. jur. Joachim Wuermeling LL.M., Honorarprofessor an der Universität Potsdam, ehem. Mitglied des Europäischen Parlaments und stv. Mitglied des Konvents zur Zukunft Europas, Mitglied des Vorstands der Deutschen Bundesbank, Frankfurt.

Der Beitrag gibt die persönliche Meinung des Autors wieder.

1 Ursula von der Leyen: Eine Union, die mehr erreichen will. Meine Agenda für Europa. Politische Leitlinien für die künftige Europäische Kommission 2019-2024, S. 23, abrufbar unter: https://ec.europa.eu/info/sites/info/files /political-guidelines-next-commission_de.pdf (letzter Zugriff: 6.4.2021).

2 Rat der Europäischen Union: Konferenz über die Zukunft Europas - Gemeinsame Erklärung, 1. März 2021, Dok. 6567/21.

3 Europäischer Rat: Erklärung von Laeken zur Zukunft der Europäischen Union, 15. Dezember 2001, S. 6, abrufbar unter: http://european-convention.europa.eu/pdf/LKNDE.pdf (letzter Zugriff: 6.4.2021).

4 Umfassend zu dem Konvent: Malte Beyer: Der Konvent zur Zukunft Europas, Baden-Baden 2007; Olaf Leiße/ Peter Becker: Die Zukunft Europas: Der Konvent zur Zukunft Europas, Wiesbaden 2005; zur Arbeitsweise: Joachim Wuermeling: Ein Werkstattbericht aus dem Verfassungskonvent - Impulse für die Konferenz zur Zukunft Europas, Freie Universität Berlin: Berliner Online-Beiträge zum Europarecht 125/2020.
} 
den bekanntlich nicht wegen seines Inhalts, sondern aus anderen politischen Gründen abgelehnt. Die von dem Konvent erarbeiteten Reformen jedoch fanden über den Vertrag von Lissabon fast vollständig ihren Weg in das europäische Recht.

Der damalige Konvent und die heutige Konferenz unterscheiden sich in vielerlei Hinsicht. Dennoch lohnt die Frage, welche die Erfolgsfaktoren für den bemerkenswerten Verlauf des Konvents waren. Lassen sich daraus Lehren für die Konferenz ziehen? Und vor allem: Welche Weichen müssen jetzt gestellt werden, damit auch die Konferenz zum Erfolg geführt werden kann? In den folgenden Ausführungen werden die Erfahrungen aus dem Konvent den Festlegungen für die Konferenz gegenübergestellt und daraus fünf Empfehlungen abgeleitet. Betrachtet werden dabei das Mandat der Konferenz, die Zusammensetzung des Gremiums und die Organisation der Beratungen.

\section{Das Mandat}

\section{Europapolitische Rahmenbedingungen}

Der Ausgangslage von Konvent und Konferenz gemeinsam ist ein hoher europapolitischer Problemdruck: Vor dem Konvent hatte die EU zwar mit der Vollendung des Binnenmarktes, der Schaffung der gemeinsamen Währung und dem Beschluss der Aufnahme der mittel- und osteuropäischen Staaten große Fortschritte erzielt. Jedoch war nun das Integrationsprogramm erschöpft. Für die Zukunft fehlte es an Orientierung und ein innerer Reformstau hatte sich aufgebaut.

Vor der Konferenz blickt die EU auf schwierige Krisenjahre zurück: Prägend waren die Finanz- und Schuldenkrise (2008, 2011), die Flüchtlingskrise (2015/2016), der Brexit (2019) und seit Anfang 2020 die COVID-19-Pandemie. Neuen globalen geopolitischen und geoökonomischen Herausforderungen scheint die Europäische Union (EU) im Auge vieler Beobachter nicht gewachsen zu sein. Zugleich warten mit der Digitalisierung und dem Klimaschutz große europäische Aufgaben.

Vor dem Konvent war die EU integrationspolitisch in die Defensive geraten: Das Unbehagen gegenüber europäischen Entscheidungen war spürbar gewachsen. Die Beteiligung an den Europawahlen erreichte 1999 mit 44,4 Prozent einen Tiefpunkt. Die drei Anläufe für Reformen mit den Verträgen von Maastricht 1992, Amsterdam 1997 und Nizza 2001 wurden als unzureichend empfunden. Eine tiefe Europaskepsis hatte sich breitgemacht.

Demgegenüber befindet sich die EU vor der Konferenz, trotz aller gegenwärtigen Kritik an den Anti-Corona-Maßnahmen, eher in einer Offensivposition. An den Europawahlen 2019 beteiligten sich über 50 Prozent der Wahlberechtigten. Anti-Europa-Bewegungen finden trotz eines weiterbestehenden Populismus von rechts und links weniger Widerhall. ${ }^{5}$ Laut einer Eurobarometer-Umfrage von $2020^{6}$ haben nur noch 20 Prozent der Bürger ein negatives Bild von der EU. Ein „verändertes Selbstbewusstsein“ stehe hinter einer EU, die „ehrgeiziger“" geworden ist, resümierte jüngst „Die Zeit“"

5 The Economist: Why leave the EU, when you can shape it instead - Eurosceptics change their tactics, 13. März 2021.

6 Europäische Kommission: Die öffentliche Meinung in der Europäischen Union, Standard-Eurobarometer 93, Sommer 2020, S. 10.

7 Matthias Krupa: Das Machtbündnis, in: Zeit Online, 29. Dezember 2020. 
Eine Herausforderung für die Konferenz dürfte eher sein, dass die Bürger von der EU mehr erwarten, als sie zu leisten imstande ist, ${ }^{8}$ als dass sie wie vor dem Konvent kaum noch Erwartungen an die EU haben.

In der Erklärung von Laeken wurden die damaligen Defizite deutlich offengelegt. Damit wurden die für den Konvent relevanten Problemkreise identifiziert und eingeengt. An einer entsprechenden, das Mandat der Konferenz eingrenzenden Diagnose fehlt es jedoch bisher.

Die Topoi des Konvents waren geprägt von der Lösung offensichtlicher Unzulänglichkeiten; so gesehen war das Ambitionsniveau - das Abstellen von Missständen - eher niedrig. Hingegen geht die Konferenz, so scheint es, mit zukunftsgewandtem Elan, mit umfassendem Gestaltungswillen und mit offenem Ehrgeiz ans Werk.

\section{Mandatsgeber}

Für den Konvent war allein der Europäische Rat Mandatsgeber. Demgegenüber wurde das Mandat der Konferenz von Europäischem Rat, Parlament und Kommission gemeinsam beschlossen. Damit gibt der Europäische Rat seine intergouvernementale Prärogative auf. Die Konferenz bekommt auf diese Weise eine breitere Legitimationsgrundlage, eine stärker supranationale, aber auch eine interinstitutionelle Prägung. Letzteres birgt die Gefahr, dass sie weniger von der offenen Debatte unter den Mitgliedern als mehr von einem Tauziehen der Institutionen geprägt sein wird.

\section{Auftrag}

Weder war der Konvent noch wird die Konferenz ein repräsentatives Beschlussgremium. Beide sollen Entscheidungen anderer vorbereiten. Im Mittelpunkt des Arbeitsauftrags von Laeken stand deshalb weniger ein fertiges Produkt, sondern eine Debatte. Erstellt werden sollte ein „Abschlussdokument [Hervorhebung durch den Autor], das entweder verschiedene Optionen mit der Angabe, inwieweit diese Optionen im Konvent Unterstützung gefunden haben, oder - im Falle eines Konsenses - Empfehlungen enthalten kann".9 Dabei stand die Diskussion der Mitglieder des Konvents untereinander im Fokus, weniger die Aufnahme von Impulsen von außen.

Die Organisation einer breit angelegten, europaweiten Debatte dominiert hingegen die Konferenz. Dabei wird die Rolle der Konferenz womöglich auf die eines bloßen Resonanzraums für externe Beiträge beschränkt. Die Diskussion im Gremium selbst tritt hingegen in den Hintergrund. Es ist bezeichnend, dass die Gemeinsame Erklärung die Arbeit in der Konferenz selbst überhaupt nicht adressiert. Zwar sollte auch der Konvent ein „Forum“ für die Zivilgesellschaft einrichten; der wesentliche inhaltliche Beitrag wurde jedoch vom Konvent selbst erwartet.

Am Ende des Konvents sollten wie auch immer geartete Empfehlungen stehen. Das Überraschende bei dem Konvent war allerdings, dass er selbst gleich zu Beginn ein ganz anderes, viel weitergehendes und konkreteres Arbeitsziel postuliert hat: die Formulierung eines Verfassungsvertrags. Mit dieser Chuzpe stellte der Konvent plötzlich ein „Produkt“

8 Hartmut Kaelble: Mehr als ein neues Krankheitsbild: Warum die Corona-Krise für die Europäische Union historisch neuartig ist, in: integration 4/2020, S. 325-332, hier S. $326 f f ., 332$.

9 Europäischer Rat: Erklärung von Laeken, 2001, S. 7. 
mit einem klar definierten Inhalt in den Raum. Wenig überraschend war, dass der eigentliche Mandatsgeber, der Europäische Rat, dies nur zähneknirschend hingenommen hat. ${ }^{10}$

Die Definition eines Produkts für die Konferenz steht noch aus. In der Gemeinsamen Erklärung ist lediglich davon die Rede, dass „über die Ergebnisse der zahlreichen im Kontext der Konferenz durchgeführten Tätigkeiten Bericht zu erstatten ist". ${ }^{11}$ Empfehlungen werden offenbar gar nicht von der Konferenz selbst, sondern von den abzuhaltenden Panels erwartet.

Im Mandat für den Konvent waren vier konkrete institutionelle Themen abgesteckt. Demgegenüber enthält die Gemeinsame Erklärung zu der Konferenz keinerlei inhaltliche Einschränkungen. Es sollen Ideen debattiert werden, „ohne dass der Gegenstand auf vorab bestimmte Politikbereiche beschränkt wird“" ${ }^{12}$

Waren also die Themenkomplexe des Konvents deutlich abgegrenzt, bleibt das inhaltliche Spektrum für die Konferenz bislang bewusst ohne jede Kontur. Für den stark partizipativen Ansatz der Konferenz mag das konsequent sein. Für die Konferenz selbst bedeutet das aber, dass sie zu einem bestimmten Zeitpunkt um eine schmerzliche Priorisierung nicht herumkommen wird.

Während dem Konvent kaum Vorgaben für die Eigenorganisation gemacht wurden, gibt die Gemeinsame Erklärung der Konferenz die Governance detailliert vor, bis hin zu Lenkungsausschüssen und Sitzungsrhythmen. ${ }^{13}$ Einer der wichtigsten Erfolgsfaktoren des Konvents war, dass dieser gleich zu Beginn vom Ende her gedacht wurde. Dazu gehörte vor allem die frühe Festlegung des Liefergegenstandes. Die Arbeiten wurden zielgerichtet so aufgesetzt, dass am Ende ein ausformuliertes Verfassungsdokument verabschiedet werden konnte. Dabei kam dem Konvent zugute, dass er über die Organisation seiner Arbeiten selbst entscheiden konnte.

Der Konferenz sollte etwas Ähnliches gelingen. Eine Idee wäre, in vielleicht neuartiger Weise ein „Mission Statement“ für die EU zu formulieren. Nachdem bisher in Verträgen, Gesetzgebung, Beschlüssen und Programmen zumeist von Zielen, Kompetenzen und Einzelmaßnahmen die Rede ist, könnten europäische Gemeinschaftsgüter festgelegt werden, die die EU künftig für die europäischen Bürger bereitstellen soll. Solche Güter könnten z. B. sein: digitale Souveränität, Klimaneutralität, Sicherheit der Außengrenzen, finanzielle Autonomie, Schutz vor europaweiten Gesundheitsbedrohungen etc. Wie diese Güter hergestellt werden sollen und was dafür institutionell erforderlich ist, kann angedeutet, muss aber nicht im Einzelnen ausbuchstabiert werden.

Empfehlung Nr. 1: Die Konferenz sollte gleich zu Beginn ein gemeinsames Verständnis dafür entwickeln, was ein Abschlussdokument konkret beinhalten soll. Auch muss frühzeitig eine inhaltliche Fokussierung erfolgen. Sonst wird man zum Schluss kaum mehr in der Lage sein, die Enden zusammenzubinden. Darauf hinwirkend muss sich die Konferenz jetzt die nötige Freiheit nehmen, ihre Aktivitäten so zu strukturieren, dass sie das gewünschte Ergebnis auch erarbeiten kann.

10 Wuermeling: Werkstattbericht, 2020, S. 11-12, m. w. N.

11 Rat der Europäischen Union: Konferenz über die Zukunft Europas, 2021, S. 4.

12 Ebenda.

13 Ebenda, S. 3-4. 
Leider setzt das nun verabschiedete Mandat vor diesem Hintergrund genau die falschen Schwerpunkte. Wo inhaltlich eine Eingrenzung sinnvoll wäre, gibt es keinerlei Grenzen. Stattdessen werden genau Vorgaben für Organisation und Ablauf gemacht, obwohl die Konferenz gerade hier Eigenständigkeit bräuchte. Metaphorisch gesprochen: Man sagt dem Architekten nicht, was er eigentlich bauen soll, aber genauestens, wie er dies zu tun hat.

\section{Das Gremium}

\section{Mitglieder}

Der Konvent hatte eine überschaubare Größe von 105 Mitgliedern und einer gleichen Zahl von Stellvertretern, Präsidentschaft und Beobachtern eingeschlossen. Werden die eigentlichen Mitglieder - ohne Präsidium und Beobachter - betrachtet, war der Konvent klar von der nationalen Seite dominiert: Er bestand zu 70 Prozent aus nationalen (Regierungen und Parlamente) und nur zu 30 Prozent aus europäischen (Parlament und Kommission) Vertretern. Allerdings teilten viele nationale Vertreter die europäische Ambition etwa der Kommissare und Europaabgeordneten. Insbesondere die nationalen Parlamentarier waren zumeist proeuropäisch gesinnt. So war der Konvent weder geprägt von dem Widerstreit nationaler Interessen noch von institutionellen Gegensätzen, sondern von einem gemeinsamen Ringen um die beste europäische Lösung, die von einer breiten Mitte getragen werden kann. Die Konferenz besteht hingegen aus über 400 Mitgliedern, die Hälfte davon sind Mitglieder des Europäischen Parlaments oder nationaler Parlamente. Neben den Vertretern der Regierungen und anderer EU-Institutionen werden auch 108 Bürger dem Plenum angehören.

Der Konvent war hochrangig besetzt, vonseiten der Mitgliedstaaten zumeist mit den Außen- und Europaministern. Der Präsident und seine Vertreter waren frühere Staatspräsidenten und Regierungschefs. Von den Mitgliedern hatten 40 Regierungsämter innegehabt, ein Dutzend waren Regierungschefs, EU-Kommissare oder Außenminister gewesen. Dies ermöglichte nicht nur eine hohe Professionalität, sondern verlieh dem Konvent auch Autorität. „So viel rhetorische Kraft, intellektueller Scharfsinn und politisches Geschick sind wohl kaum für ein europäisches Projekt aufgewendet worden“, wurde damals resümiert. ${ }^{14}$

Weder der Konvent noch die Konferenz sind repräsentativ zusammengesetzt. Beide vermitteln keine demokratische Legitimation. Im Konvent ergaben sich Einfluss und Verhandlungsmacht eher aus der Überzeugungskraft und Autorität der Persönlichkeit als aus der Machtposition der entsendenden Institution.

\section{Leitung}

Der Konvent wurde straff von oben geführt. Alle maßgeblichen Entscheidungen wurden letztlich auf Vorschlag des Präsidenten vom Präsidium getroffen und dem Plenum nur zur Billigung vorgelegt. Es hat keine einzige Abstimmung gegeben. Prägend war die starke und dominierende Rolle von Valéry Giscard d'Estaing als Präsident des Konvents. Seine Methode bestand allerdings keineswegs in einem autoritären und präsidialen „Durchregieren“, wie befürchtet worden war. Natürlich hat Giscard d'Estaing auch hart durchgegriffen,

14 Wuermeling: Werkstattbericht, 2020, S. 41. 
wenn es ihm nötig schien. Aber er hat eben auch stundenlang regelrecht stoisch den Debatten zugehört und Anregungen aufgenommen. Giscard d'Estaing konnte hohe Integrationskraft entfalten, weil er sich inhaltlich ergebnisoffen und neutral gab. Seine Ideen entwickelten sich "gemeinsam mit dem Konvent während des Fortgangs der Arbeiten“, wird er zitiert. ${ }^{15}$ Einen glasklaren Plan aber hatte Giscard d'Estaing von dem Ablauf, ja von der Dramaturgie des Konvents und eine ebenso präzise Vorstellung von dem formalen Ergebnis, einem ausformulierten Verfassungsvertrag.

Für die Konferenz konnten sich die Institutionen entgegen den Vorstellungen des Rates nicht auf die Berufung eines Präsidenten verständigen; sie wird vielmehr von einem "gemeinsamen Vorsitz" ${ }^{16}$ einem dreiköpfigen Gremium von Repräsentanten der drei Institutionen, geleitet. Eine solche Aufstellung birgt große Risiken. Eine strategische Führung der Konferenz ist von einem solchen Triumvirat kaum zu erwarten, denn es ist weder unabhängig noch neutral. So ist zu befürchten, dass die Dreier-Präsidentschaft die Konferenz wenig planvoll führt, sondern sich in interinstitutionellen Machtkämpfen, Auseinandersetzungen und Rangeleien verliert. Schon ist schlicht von einem „Trilog "17 die Rede. Die Konferenz hat mithin keine eindeutige Führung, kein „Gesicht" und auch keine durch eine Persönlichkeit vermittelte Autorität, weder nach innen noch nach außen. Das ist für die Konferenz eine echte Hypothek. Zu hoffen bleibt, dass sich trotz der Dreierleitung eine Persönlichkeit durch Unvoreingenommenheit, Neutralität und Unabhängigkeit auszeichnet und sich als integrierende Führungskraft etabliert.

Das zehnköpfige Präsidium des Konvents war klein genug, um ergebnisorientiert arbeiten zu können, und gleichzeitig groß genug, um die großen Strömungen zu repräsentieren. Die Tagungsfrequenz war hoch, die Beratungen waren intensiv und gingen bis ins Detail. Dort wurde das vielfältige Material der Papiere der Mitglieder und Arbeitsgruppen und -kreise zu dem Verfassungsvertrag verarbeitet. Es gab heftige Auseinandersetzungen, die zum Teil in der Öffentlichkeit ausgetragen wurden. Die Ergebnisse zeichneten sich dennoch durch Ambition und Mut aus. Zugleich gelang es, eine fein austarierte politische Balance zu erzielen, die auf Zustimmung im Konvent und später bei den Regierungen traf. Dafür war entscheidend, dass das Präsidium gegenüber dem Plenum trotz aller Meinungsverschiedenheiten geschlossen auftrat.

In der Konferenz gibt es unterhalb des gemeinsamen Vorsitzes einen von den Institutionen paritätisch besetzten, neunköpfigen „Exekutivausschuss“, der „einvernehmlich“ entscheiden muss. ${ }^{18}$ Die drei Institutionen repräsentieren aber keineswegs zwangsläufig die zu erwartenden inhaltlichen Richtungen oder gar Lager; die Trennlinien dürften jedenfalls bei Rat und Parlament innerhalb der Institution verlaufen. So dürfte es dem Dreiergremium schwerfallen, Positionen für das Plenum zu einem Konsens zu aggregieren.

Empfehlung Nr. 2: Die Konferenz sollte zielorientiert geführt werden. Dies steht nicht im Widerspruch zu dem partizipativen Anspruch und der Ergebnisoffenheit. Im Gegenteil be-

15 Ebenda, S. 33

16 Rat der Europäischen Union: Konferenz über die Zukunft Europas, 2021, S. 4.

17 Nicolai von Ondarza/Minna Ålander: Die Konferenz zur Zukunft Europas. Herausforderungen und Erfolgsbedingungen für einen Reformimpuls, der über Krisenmanagement hinausreicht, Stiftung Wissenschaft und Politik: SWP-Aktuell 20/2021, S. 4.

18 Rat der Europäischen Union: Konferenz über die Zukunft Europas, 2021, S. 4. 
darf es gerade bei der Einbeziehung vieler Anspruchsgruppen und Themen einer Leitung, die steuern, ordnen und fokussieren kann.

\section{Gliederung}

Die Mitglieder des Konvents hatten sich in einer Matrixform organisiert. Einerseits bildeten sie die „Komponenten“ nationale Parlamente, nationale Regierungen, Europäisches Parlament und Europäische Kommission; zugleich aber formten sie parteipolitische Fraktionen und thematische Gruppen. Während die Komponenten mangels gemeinsamer Interessen kaum Einfluss gewinnen konnten, entwickelten sich die Fraktionen zu den prägenden Kräften. ${ }^{19}$ Viele kleine informelle Gruppen, z. B. die „Freunde der Subsidiarität“, hatten sich einzelnen Anliegen verschrieben und konnten punktuelle Erfolge erzielen.

Empfehlung Nr. 3: In der Konferenz sollte die Dysfunktionalität der Konventsgliederung - das Auseinanderfallen von formaler Stellung und politischer Bedeutung von Untergliederungen - vermieden werden. Die rein institutionelle Zugehörigkeit vermag angesichts der inneren Heterogenität kein ausreichendes „Ownership“ zu vermitteln. Die Mitglieder der Konferenz sollten sich vielmehr in möglichst homogenen Gruppen organisieren, um die Meinungsbildung über die Untergliederungen kanalisieren zu können. Im Konvent haben sich die politischen Fraktionen zu diesem Zweck als besonders geeignet erwiesen.

\section{Das Vorgehen}

\section{Zeitplan}

Der Konvent tagte 17 Monate, von Februar 2002 bis Juli 2003. Das Plenum hielt insgesamt 52 Sitzungstage ab. Der Zeitraum erscheint im Rückblick lang genug für eine gründliche Arbeit und zugleich kurz genug, um das Momentum zu erhalten und Einigungsdruck zum Ende hin zu erzeugen.

Die Konferenz ist nur für ein knappes Jahr angesetzt. Zunächst soll ein intensives Programm an Bürgerdialogen stattfinden. Viel Zeit bleibt dann nicht mehr, um substanzielle Ergebnisse zu erarbeiten, die über die Zusammenfassung des Gehörten hinausgehen. Unklar bleibt, welche Rolle das Plenum der Konferenz spielen soll, wenn es nach der Gemeinsamen Erklärung nur mindestens einmal im Halbjahr zusammentreten soll.

\section{Ablauf}

Der Konvent verständigte sich frühzeitig darauf, seine Beratungen in drei Abschnitte zu gliedern. Während in der ersten Etappe so viel Stoff wie möglich gesammelt, das „Konventsmaterial“ in extenso ausgeweitet wurde, hatte die zweite Etappe die Funktion eines Filters und führte zu einer drastischen Reduzierung des Materials, das dann in der letzten Phase in Verfassungsartikel gegossen wurde.

In der viermonatigen ersten „Phase des Zuhörens“ wurde die Debatte breit geöffnet, sowohl innerhalb des Konvents als auch nach außen. In sechs Plenartagungen mit einem „Stakkato" von dreiminütigen Wortbeiträgen ${ }^{20}$ wurde die gesamte Palette der Reformthe-

19 Wuermeling: Werkstattbericht, 2020, S. $27 \mathrm{ff}$.

20 Ebenda, S. 12. 
men in fünf thematischen Abschnitten erörtert. Die Mitglieder und Stellvertreter reichten dazu 400 „schriftliche Beiträge“ ein. Dabei wurde „[j]eder nur denkbare Aspekt (...) in irgendeiner Form “21 vorgetragen. Bei einem zweitägigen Forum der Zivilgesellschaft kamen 160 Organisationen zu Wort. Die Beiträge waren insgesamt wenig ergiebig, in der Sache erwartbar und von dem Partikularinteresse der Petenten bestimmt. Gut 200 junge Leute erarbeiteten auf einem Jugendkonvent eine Entschließung an den Konvent. Die Erfahrung lehrte hier, dass die Bürger mit mittel- und langfristigen politischen Zukunftsplanungen in der Europapolitik gemeinhin wenig anfangen können. Die Fähigkeit zur klaren Positionierung und Argumentationsstärke sind eher Interessengruppen und extrovertierten Persönlichkeiten zu eigen.

Für die zweite „Phase der Diskussion“ von sechs Monaten wurde das Material auf Themenblöcke verteilt und in 14 Gruppen von 30 bis 60 Konventsmitgliedern in fünf bis zehn Sitzungstagen diskutiert. Dem Plenum wurden auf dieser Grundlage Vorschläge für die Formulierung des Verfassungsvertrages präsentiert. Zwischen Februar und Juli 2003, in der dritten „Phase der Redaktion“, wurden die Texte des Verfassungsvertrags formuliert, zunächst in sechs Paketen, und zuletzt in ihrer Gesamtheit diskutiert. Die Konventsmitglieder nutzten intensiv die Gelegenheit, in kurzer Frist Änderungsanträge zu den Entwürfen zu stellen. Es gingen 7000 Anträge ein, die im Plenum diskutiert, aber vom Präsidium beschieden wurden. Abstimmungen zu einzelnen Anträgen gab es mit Hinweis auf die fehlende Repräsentativität des Konvents nicht. Bemerkenswert war in dieser Phase die erhebliche Zunahme des Gewichts der Mitgliedstaaten. Denn das Präsidium wollte einen Text vorlegen, der als Ganzes in den Regierungen konsensfähig war. So wurde vielfach auf nationale Einzelinteressen Rücksicht genommen, deren Nichtberücksichtigung zum „Showstopper“ hätte werden können. Das Paket sollte so ausbalanciert sein, dass keiner es mehr aufschnüren mochte, aber doch so ambitioniert, dass es einen echten Fortschritt mit sich brachte.

Für die Konferenz wird derzeit ausschließlich über den Teil diskutiert, der im Konvent der Phase des Zuhörens entsprach, der Sammlung von Impulsen von Bürgern sowie Vertretern der Zivilgesellschaft, ${ }^{22}$ obwohl sich die EU mit der partizipativen Demokratie bislang schwergetan hat. ${ }^{23}$ Dazu sollen nach der Gemeinsamen Erklärung klassische Veranstaltungen stattfinden, aber vor allem auf interaktiven, mehrsprachigen digitalen Plattformen debattiert werden. ${ }^{24}$ Per Zufall ausgewählte Bürger werden zu „Bürgerforen“ gebeten, die im weiteren Verlauf Feedback zu der Behandlung ihres Themas geben. ${ }^{25}$ Der Ehrgeiz besteht darin, so die Gemeinsame Erklärung, „weit über die Hauptstädte Europas hinaus alle Ecken der Union“ ${ }^{26}$ zu erreichen und „einen neuen Raum für die Debatte mit den Bürgerinnen und Bürgern“ zu eröffnen. ${ }^{27}$

21 Ebenda, S. 14.

22 Europäische Kommission: Mitteilung der Kommission an das Europäische Parlament und den Rat. Gestaltung der Konferenz zur Zukunft Europas, COM(2020) 27 final, S. 4-7; Europäisches Parlament: Standpunkt des Europäischen Parlaments zur Konferenz über die Zukunft Europas, 15. Januar 2020, P9_TA(2020)0010, Nr. 412.

23 Von Ondarza/Ålander: Die Konferenz zur Zukunft Europas, 2021, S. 6.

24 Rat der Europäischen Union: Konferenz über die Zukunft Europas, 2021, S. 3-4.

25 Europäisches Parlament: Standpunkt zur Konferenz über die Zukunft Europas, 2020, Nr. 11.

26 Rat der Europäischen Union: Konferenz über die Zukunft Europas, 2021, S. 3.

27 Ebenda, S. 2. 
Die Konferenz sollte keine überzogene Erwartung an die Bürgerdialoge stellen. Das partizipatorische Ungestüm darf nicht den nüchternen Blick darauf verstellen, dass in einer pluralistischen Demokratie kein Konsens der Bürger erwartet werden kann, den erfolgreiche Politik nur „abzugreifen“ braucht. ${ }^{28}$ Betont man den vorbereitenden Charakter der Konferenz, kann sie sich in der Tat darauf beschränken, einen Ergebnisbericht über ihre Dialoge zu verfassen. Darauf scheinen die nationalen Regierungen heute zu hoffen, wie übrigens auch vor dem Konvent. Dass es aber auch anders geht, hat der Konvent gezeigt.

Empfehlung Nr. 4: Das im Bürgerdialog gesammelte Material muss fokussiert und in eine zielführende Form gebracht werden. Es muss die Chance bestehen, dass die Mitglieder ihr Potenzial entfalten können. Sollen sinnhafte Schlussfolgerungen aus den Debatten gezogen werden, braucht es dafür Zeit, aber auch Ziel und Struktur. Die Sacharbeit mag zwar weniger geneigte Aufmerksamkeit auf sich ziehen als interaktive Digitalformate, bringt aber den für die EU womöglich entscheidenden „echten europäischen Reformimpuls“.29 Für die Konferenz sollte es einen Plan jenseits der Bürgerbeteiligung geben. Die Erwartung an das Gremium sollte sein, dass es auf dieser Basis selbst neue Ideen und progressive Konzepte für die EU von morgen entwirft.

\section{Fazit}

„Wird der erste Knopf falsch eingeknöpft, schließt die Jacke immer schief“, weiß der Volksmund. Dieser Gefahr sieht sich die Konferenz aktuell gegenüber. Es drängt sich der Eindruck auf, als nutze der Rat die Erfahrungen aus dem Konvent 2002/2003 in adverser Weise, indem er der Konferenz die entscheidenden erfolgsfördernden Elemente vorenthält. Ein „Hybrid aus interinstitutionellen Verhandlungen und Bürgerkonsultation“"30 kann die hohen Erwartungen nicht erfüllen. Es kann nur an die Mitglieder appelliert werden, trotz der widrigen Umstände mit Selbstbewusstsein und Gestaltungswillen die Geschicke der Konferenz selbst in die Hand zu nehmen.

Empfehlung Nr. 5: Die Konferenz sollte jetzt vom Ende her gedacht werden. Wenn sie Europa strategisch und programmatisch voranbringen will, braucht sie eine straffe Führung, eine klare Vorstellung von dem angestrebten Ergebnis und einen zielführenden Arbeitsplan. Ein Spannungsbogen muss entstehen; Eigendynamik und Kreativität brauchen Raum zur Entfaltung.

Schöpferisches Denken bedeutet, so heißt es jüngst bei Stefan Klein, „den Möglichkeitsraum zu erweitern“. ${ }^{31}$ Entsprechend „eingeknöpft“ kann die Konferenz für die europäische Integration genau das leisten.

28 Die Kommission meint tatsächlich, herausfinden zu können, was die Bürger „wirklich wollen“, siehe Europäische Kommission: Gestaltung der Konferenz zur Zukunft Europas, 2020, S. 8.

29 Von Ondarza/Ålander: Die Konferenz zur Zukunft Europas, 2021, S. 7.

30 Ebenda, S. 6.

31 Stefan Klein: Keiner denkt für sich allein, in: Zeit Magazin, 4. März 2021. 